\title{
Scale lengths of inter-ELM fluctuations in the pedestal of ASDEX Upgrade
}

\author{
B Kurzan, C Fuchs, A Scarabosio, B D Scott and ASDEX Upgrade Team
}

\author{
Max-Planck-Institut für Plasmaphysik, EURATOM Association, \\ Boltzmannstr. 2, 85748 Garching, Germany
}

Email: Bernd.Kurzan@ipp.mpg.de

\begin{abstract}
Fluctuations of electron density and temperature in the hot pedestal are investigated in-between type-I edge localised modes (ELMs) by high resolution, high precision Thomson scattering. The observed fluctuations of electron density and temperature are correlated and in phase. The amplitudes of these fluctuations become observable above a line averaged electron density of about $4 \times 10^{19} \mathrm{~m}^{-3}$. The heat transport in the pedestal is investigated by analysing the heat diffusivity. The experimentally found variations in the relative fluctuation amplitudes of electron density and temperature and of the heat diffusivity can be explained by different scale lengths of the underlying turbulence. The large fluctuations of electron density and temperature scale with a hybrid scale between ideal and resistive magneto-hydrodynamics, while the thermal transport occurs on the smallest scale lengths of the turbulence.
\end{abstract}




\section{Introduction}

In $\mathrm{H}$ mode plasmas [1] in-between type-I edge localised modes (ELMs) [2] large scale fluctuations of the electron density and temperature were found recently in the middle of the pedestal on ASDEX Upgrade [3]. They appear in 2D poloidal snapshots of the electron density and temperature, which are measured by high precision, high resolution Thomson scattering, as 'blobs' and 'dips' on the low field side on the closed magnetic flux surfaces inside the separatrix. The fluctuating structures in the edge plasmas investigated in [3] often show quasi-periodic structures. With the assumption that the fluctuations are field-aligned, toroidal quasi-mode numbers were derived. Fluctuations of the electron density were already observed with reflectometry in the steep edge gradient region inside the separatrix [4]: The radial correlation lengths, which were found for L mode plasmas and plasmas near the L-H threshold, scaled as expected for drift-resistive ballooning turbulence. For $\mathrm{H}$ mode plasmas the radial correlation lengths scaled with the ion sound gyro-radius.

Fluctuations in the steep edge gradient region and in the scrape-off layer (SOL) were also investigated for $\mathrm{H}$ mode plasmas on other machines: On Alcator C-Mod data of Ohmic $\mathrm{L}$ and $\mathrm{H}$ mode edge plasmas were compared with the predictions of turbulence theories [5]. Filaments in-between ELMs, which propagate in the SOL, were found recently on MAST [6].

In this paper a set of $\mathrm{H}$ mode plasmas with parameters covering a broad range are analysed to find scalings for the observed fluctuations. A key parameter in the scalings is the electron density: The relative fluctuations in the pedestal are larger than the error of measurement above a line averaged electron density of about $4 \times 10^{19} \mathrm{~m}^{-3}$ and increase with electron density. The relative fluctuation amplitudes, however, do not only depend on the electron density, but also on other parameters, as will be shown. Additionally the heat transport is investigated by analysing the heat diffusivity in the pedestal. The experimentally found variations in the relative fluctuation amplitudes of electron density and temperature, and in the heat diffusivities, correlate with different scale lengths of the underlying turbulence: The fluctuating structures of electron density and temperature have large scale lengths, while the thermal transport occurs on the smallest scale lengths of the turbulence.

This paper is organised in the following way: In section 2 the experimental setup is explained, followed by a presentation of the experimental results in section 3 . The scale lengths of the radial diameters of the fluctuating structures of electron density and temperature, and of the heat diffusivity, will be compared with the scale lengths used in simulations of the drift-Alfvén turbulence [7] in section 4.

\section{Experimental Setup}

With the vertical Thomson scattering diagnostic [8] electron density and temperature profiles are measured at ASDEX Upgrade with high accuracy. The light source consists of up to $6 \mathrm{Nd}$-YAG lasers. The Thomson-scattered light is imaged directly through air onto 16 polychromator channels defining 16 scattering volumes in the 
(a)
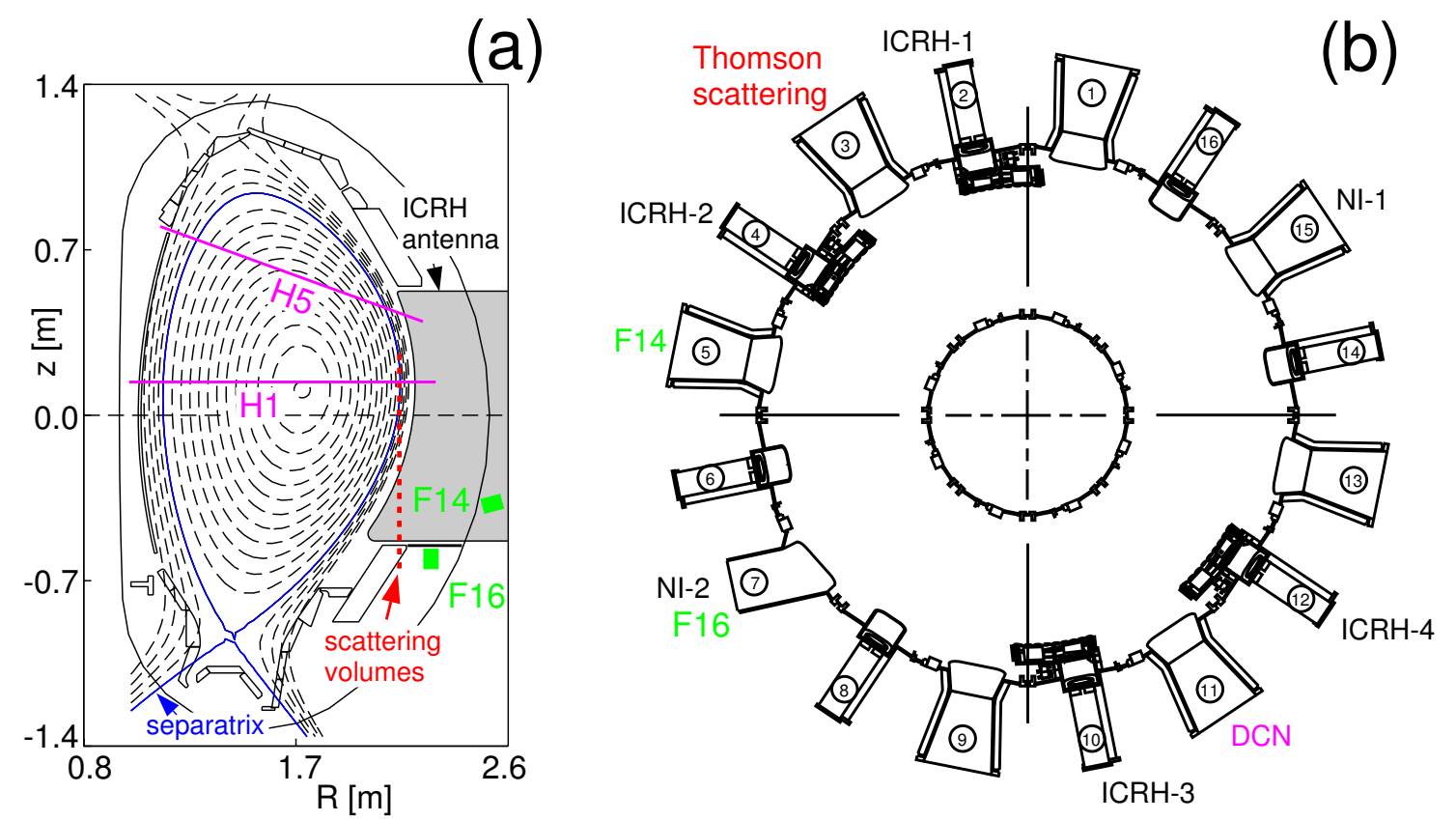

Figure 1: Poloidal cross section of the ASDEX Upgrade vessel (a) with the DCNinterferometer line integrals $H 1$ and $H 5$, the ionisation gauges $F 14, F 16$, and the scattering volumes of the edge Thomson scattering diagnostic. The diagnostics have different toroidal positions as can be seen in the horizontal cut of the torus (b).

plasma. By shifting the whole diagnostic either core, or edge profiles are measured. At the plasma edge 10 of the 16 scattering volumes are available (see figure 1 (a)). A single scattering volume has a length of $25 \mathrm{~mm}$ in the vertical direction and a diameter of about $1.5 \mathrm{~mm}$, which is the laser beam's diameter. The lasers are stacked in the radial direction with a distance of $2.7 \mathrm{~mm}$ between adjacent lasers. With improved data acquisition and evaluation [9] high precision electron density and temperature data are obtained. The relative statistical errors for electron densities $n_{e} \geq 5 \times 10^{18} \mathrm{~m}^{-3}$ are now $\delta n_{e, s t a t} / n_{e} \leq 10 \%$ and $\delta T_{e, s t a t} / T_{e} \leq 15 \%$. Systematic errors of the Thomson scattering diagnostic are still present although not completely understood. From the channel-to-channel variation of assumed-to-be smooth profiles relative systematic errors of $\delta n_{e, s y s} / n_{e} \approx 7 \%$ and $\delta T_{e, s y s} / T_{e} \approx 7 \%$ are estimated.

The edge Thomson scattering data are compared with the signals of other diagnostics: a) The line integrals of the electron density, divided by the integration path length are measured by the DCN-interferometer. The line density $\left\langle n_{e}\right\rangle_{H 1}$ has an integration path through the plasma centre, and the line density $\left\langle n_{e}\right\rangle_{H 5}$ covers the plasma edge including the pedestal shoulder. b) The neutral particle fluxes at the outer midplane and in the pumping chamber, $f_{m p l}$ and $f_{p c h}$, are measured by the ionisation gauges F14 and F16 respectively. The poloidal and toroidal positions of these diagnostics are shown in figures $1(\mathrm{a})$ and $1(\mathrm{~b})$. The head of the ionisation gauge of the so-called ASDEX-type [10] is installed in a box with a small entrance hole and measures a neutral particle density, which is usually different from the external density. Through proper calibration the gauge signal can be related to the 
Table 1: Plasma parameters.

\begin{tabular}{l|l}
\hline toroidal magnetic field & $-3.0 \mathrm{~T} \leq B_{t} \leq-1.9 \mathrm{~T}$ \\
plasma current & $I_{p l} \in\{0.8 \mathrm{MA}, 1.0 \mathrm{MA}\}$ \\
line averaged electron density & $4.6 \leq\left\langle n_{e}\right\rangle_{H 1}\left[10^{19} \mathrm{~m}^{-3} \leq 9.3\right.$ \\
heating power by neutral beam co-injection & $2.6 \mathrm{MW} \leq P_{N B I} \leq 13.2 \mathrm{MW}$ \\
heating power by ion cyclotron heating & $1.2 \mathrm{MW} \leq P_{I C R H} \leq 3.2 \mathrm{MW}$ \\
elongation & $1.34 \leq \kappa \leq 1.84$ \\
lower triangularity & $0.31 \leq \delta_{l} \leq 0.55$ \\
upper triangularity & $-0.07 \leq \delta_{u} \leq 0.50$ \\
\hline
\end{tabular}

neutral flux density outside the gauge without any assumption on the velocity distribution of the neutrals, if the conductance into the ionisation volume is sufficiently small [10].

\section{Results}

The data, which are analysed in this paper, are taken from the plateau phases of 49 type-I ELMy $\mathrm{H}$ mode discharges in deuterium, and collected in a data base. They cover the parameter range summarised in table 1.

\subsection{Large scale fluctuations in $1 \mathrm{D}$ radial profiles}

The statistical error of measurement for electron density and temperature determined by Thomson scattering is so small that large scale plasma fluctuations are the main source for the scatter in the edge profiles. These fluctuations were analysed in detail for a selected type of $\mathrm{H}$ mode discharges in [3]: A fraction of $61 \%$ of these fluctuations showed a quasi-periodic structure in the poloidal plane. This means that in 2D poloidal snapshots of electron density and temperature, blobs and dips are located along a magnetic flux surface with roughly the same difference in poloidal angle between them. In this paper we are interested in the variation of the amplitudes of these large scale fluctuations, including both quasi-periodic and non-periodic structures, with different discharge parameters. When mapping the positions of the Thomson scattering volumes in the poloidal direction along the magnetic flux surfaces to the outer midplane, a 1D radial profile can be obtained with the major radius as the radial coordinate: The radial background profiles, unperturbed by fluctuations, can be estimated, because the positive and negative perturbations to the profiles, which were measured at different poloidal positions, are eliminated when averaging over them. For improved definition of the background profile and of the fluctuations, data can be used, which are taken during a time interval in a plateau phase, where the plasma parameters are constant.

A typical example of such a 1D radial profile is shown in figure $2(a)$. The radial coordinate is here $R-R_{\text {out }}$ with $R$ as the major radius and $R_{\text {out }}$ the position of the separatrix in the outer midplane as determined from magnetics. For this profile data points which appear at a time $t$ close to an ELM were left out, 

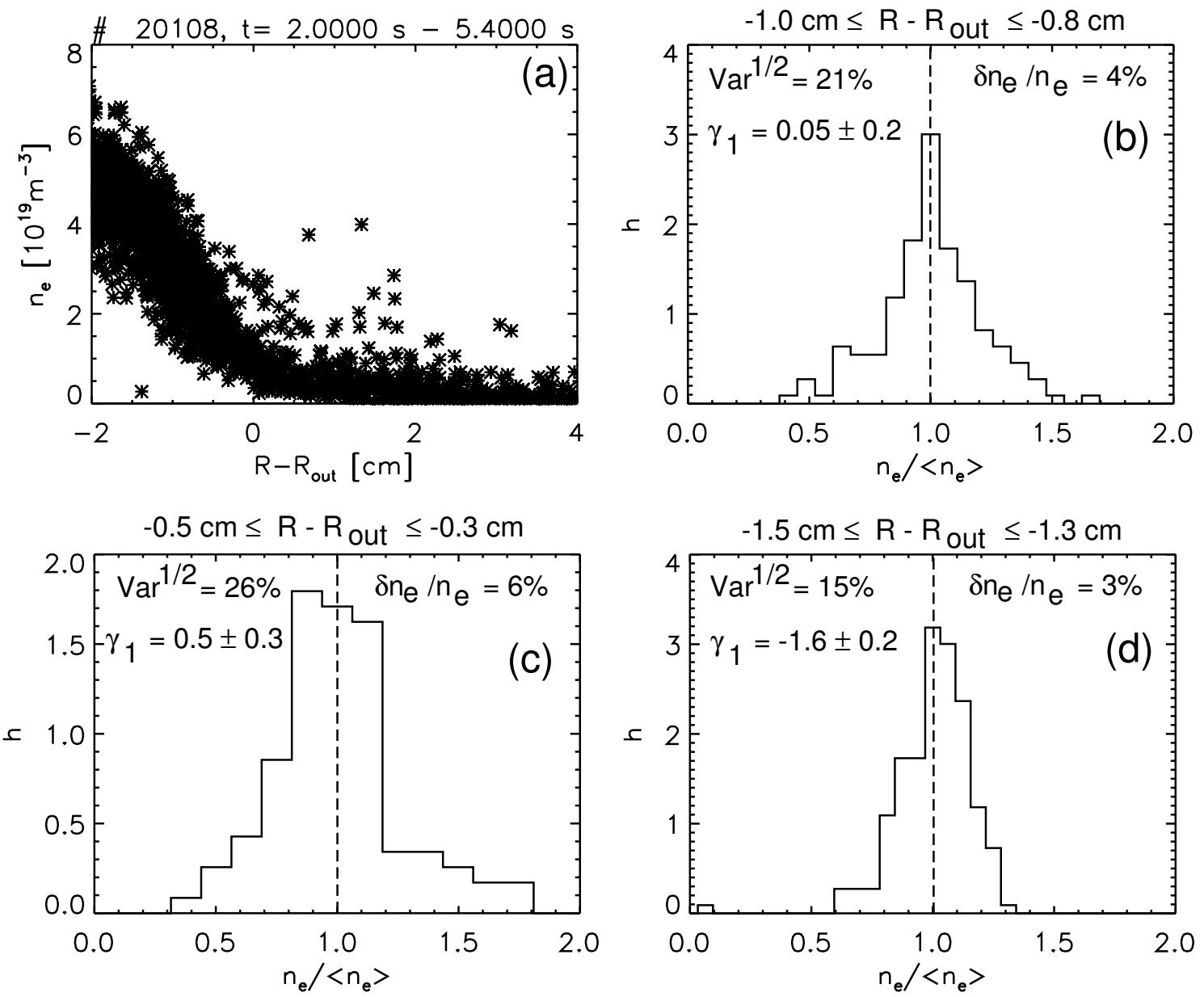

Figure 2: The $1 D$ radial electron density profiles plotted over the time interval $2 s \leq t \leq 5.4 \mathrm{~s}$ excluding the ELMs, $t \notin\left[t_{E L M}-0.5 \mathrm{~ms}, t_{E L M}+3 \mathrm{~ms}\right]$ show large scatter (a). Histograms $h$ of the electron densities $n_{e}$ normalised to the arithmetic mean $\left\langle n_{e}\right\rangle$ taken at different radial positions $R-R_{\text {out }}$, are used to characterise the fluctuations. The scatter of the normalised densities, $\operatorname{Var}^{1 / 2}$, is larger than the error of measurement $\delta n_{e} / n_{e}$. Around $R-R_{\text {out }} \approx-0.9 \mathrm{~cm}$ the histograms are nearly symmetric (skewness $\gamma_{1} \approx 0$ ) (b), further outwards the profiles are more often perturbed by maxima $\gamma_{1}>0$ (c). Further inwards it is vice versa (d). (Typical error of measurement: $\left.\Delta\left(R-R_{\text {out }}\right)=5 \mathrm{~mm}\right)$. 
$t \notin\left[t_{E L M}-0.5 \mathrm{~ms}, t_{E L M}+3 \mathrm{~ms}\right]$, with $t_{E L M}$ marking the maximum in $D_{\alpha}$ intensity during an ELM: During this specified time interval around the ELM the radial electron density and temperature profiles first flatten due to the ELM crash and then steepen again. For the discharges investigated in this paper no further evolution of the profiles was found for times, which are further away from the ELM (see also [3]). Histograms of the electron density $n_{e}$ normalised to the arithmetic mean $\left\langle n_{e}\right\rangle$ over a time interval, are plotted for several radial positions $R-R_{\text {out }}$ in figures 2 (b, c, d). The arithmetic mean values were determined for each scattering volume within the radial range of interest individually over the specified time interval where the plasma parameters were constant. Thus calibration errors do not show up in the histograms. The intervals of the histograms are set to twice the relative statistical error $\delta n_{e} / n_{e}$. The scatter of the data, $\operatorname{Var}^{1 / 2}$, with $\operatorname{Var}$ as the variance of the normalised electron densities, $\operatorname{Var}^{1 / 2}\left(n_{e} /\left\langle n_{e}\right\rangle\right)$, is larger than the error of measurement and can therefore clearly be resolved. The skewness $\gamma_{1}=\mu_{3} / \operatorname{Var}^{3 / 2}$, with $\mu_{3}$ as the third central moment describes the asymmetry of the histogram. The shape of the histogram depends on the radial position: In general, in the middle of the pedestal the histogram is symmetric $\left(\gamma_{1} \approx 0\right)$ (figure $2(\mathrm{~b})$ ). Further outwards more maxima than minima perturb the profile $\left(\gamma_{1}>0\right)$ (figure $\left.2(\mathrm{c})\right)$ and further inwards it is vice versa (figure 2(d)), see also [3]. For the comparison of the fluctuation amplitudes for different discharge parameters, the fluctuation amplitudes are always determined at the radial position $R_{\text {sym }}$, where the histogram is symmetric.

Note that not for all of the 49 investigated discharges the fluctuation amplitudes could be separated from the statistical error of the Thomson scattering diagnostic. In these cases no information about the plasma fluctuations could be obtained. So the number of data points in the following figures is usually below the number of investigated discharges.

\subsection{Radial positions of the symmetric histograms}

For understanding the radial positions where the fluctuations of electron density and temperature show symmetric histograms, one must consider that the accuracy of the separatrix position in the outer midplane, $R_{\text {out }}$, which is determined from magnetics, is only $\pm 5 \mathrm{~mm}$. This is not enough for investigating properties of the steep $\mathrm{H}$ mode edge profiles. From transport calculations it is known that at the true separatrix the electron temperature in the outer midplane is around $100 \mathrm{eV}$ [11]. So the true position of the separatrix in the outer midplane, $R_{\text {out }, 100}$, can be determined with higher accuracy from the electron temperature profile, which is measured by Thomson scattering.

Important parameters for characterising the steep gradient region at the edge, where the symmetric histograms of the fluctuations are found, are the gradient scale lengths of the electron density, $l_{n e}=\left\langle n_{e}\right\rangle /\left(d\left\langle n_{e}\right\rangle / d R\right)$, and the analogously defined gradient scale lengths of the electron temperature, $l_{T e}$, and pressure, $l_{p e}$. The radial gradient of the electron density profile, $d\left\langle n_{e}\right\rangle / d R$, and of the electron temperature and pressure, are obtained by linear fits to the respective profiles at the radial positions of the symmetric histograms. 

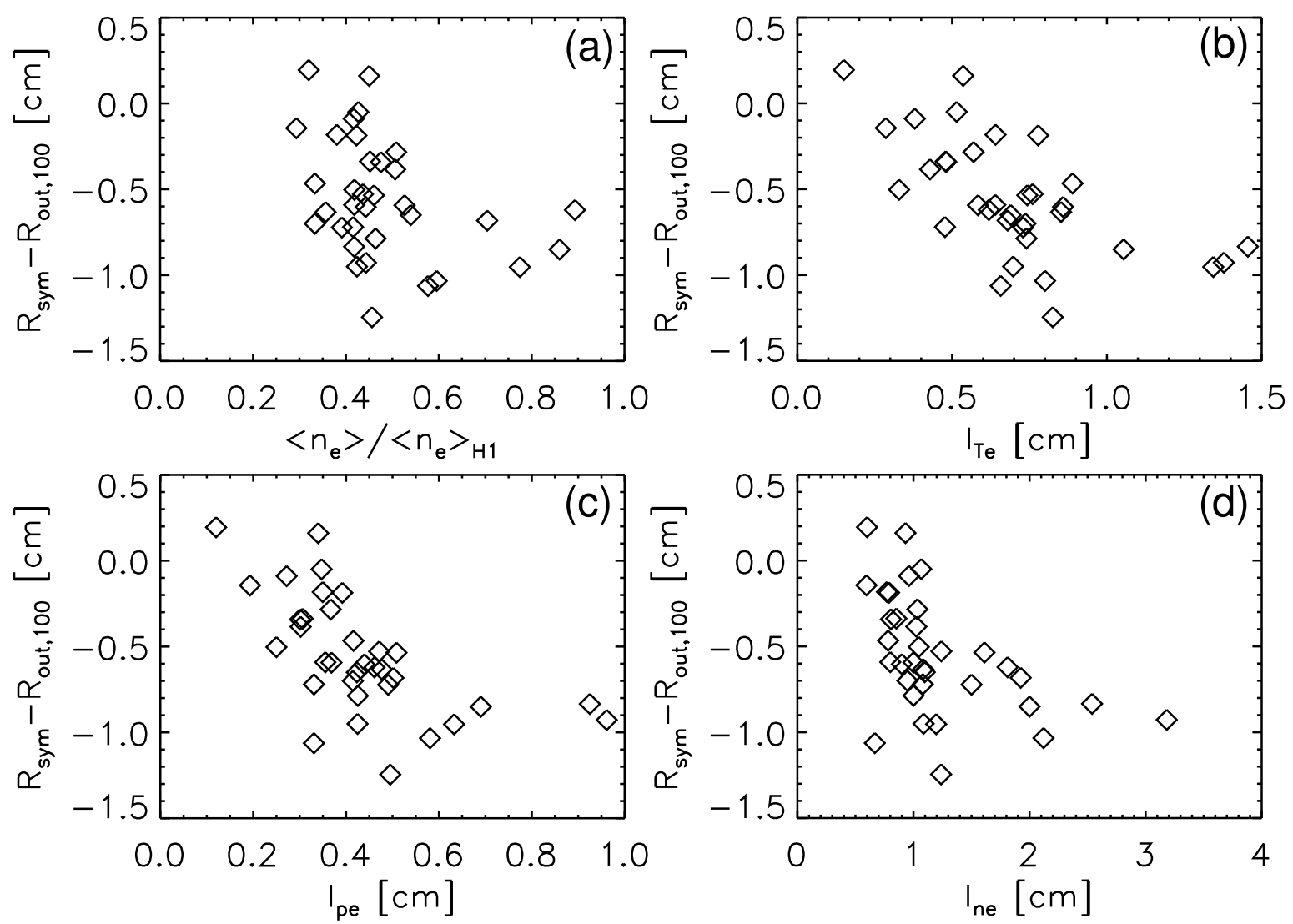

Figure 3: The symmetric histograms of the electron density and temperature fluctuations are located at radial positions $R_{\text {sym }}-R_{\text {out }, 100}$ where the time-averaged local electron density $\left\langle n_{e}\right\rangle$, normalised to the line integral $\left\langle n_{e}\right\rangle_{H 1}$, is $\left\langle n_{e}\right\rangle /\left\langle n_{e}\right\rangle_{H 1}>0.3$ (a). The radial positions $R_{\text {sym }}-R_{\text {out }, 100}$ are well correlated with the smallest gradient scale lengths occurring in the pedestal like those of electron temperature $l_{T e}$ (b) and electron pressure $l_{p e}(c)$. They are less correlated with the larger gradient scale lengths of the electron density $l_{n e}(d)$. The corresponding values of $\eta_{e}=l_{n e} / l_{T e}$ vary between $1.3 \leq \eta_{e} \leq 2.5$. (Typical errors of measurement: $\Delta\left(R_{\text {sym }}-R_{\text {out }, 100}\right) \leq 2 \mathrm{~mm}, \Delta\left(\left\langle n_{e}\right\rangle /\left\langle n_{e}\right\rangle_{H 1}\right) /\left(\left\langle n_{e}\right\rangle /\left\langle n_{e}\right\rangle_{H 1}\right)=5 \%, \Delta l_{T e} / l_{T e} \leq 10 \%$, $\left.\Delta l_{\text {pe }} / l_{\text {pe }} \leq 15 \%, \Delta l_{\text {ne }} / l_{\text {ne }} \leq 8 \%\right)$. 
The radial positions $R_{\text {sym }}$ of the symmetric histograms relative to the position of the separatrix $R_{o u t, 100}$ are found where the local electron density $\left\langle n_{e}\right\rangle$, which is averaged over the time interval of evaluation, is above $\left\langle n_{e}\right\rangle /\left\langle n_{e}\right\rangle_{H 1}>0.3$, with $\left\langle n_{e}\right\rangle_{H 1}$ as the line integral of the electron density (see figure 3(a)). This is approximately in the middle of the pedestal and further inwards. The radial positions of the symmetric histograms relative to the position of the separatrix correlate quite well with the smallest local gradient scale lengths like the electron temperature and electron pressure gradient scale lengths $l_{T e}$ and $l_{p e}$ respectively (see figures 3(b) and (c)), instead of the larger electron density gradient scale lengths $l_{n e}$ (figure $3(\mathrm{~d})$ ). So with steeper profile gradients the positions of the symmetric histograms move outwards, closer to the separatrix. The values of $\eta_{e}=l_{n e} / l_{T e}$ vary indeed between $1.3 \leq \eta_{e} \leq 2.5$ for the data shown in figure 3. A systematic study of the dependencies of $\eta_{e}$ will be the subject of a future paper.

\subsection{Correlation of electron density and temperature fluctu- ations and radial diameters}

In the $2 \mathrm{D}$ plots of the large scale fluctuations of electron density and temperature it could already be seen that the quasi-periodic structures of the large scale fluctuations of electron density and temperature are correlated and in phase [3]. This is tested now for the fluctuations observed in the poloidally averaged 1D profiles, which include additionally small amplitude quasi-periodic and non-periodic fluctuations. The relative deviations of the electron density $n_{e}$ from their mean value $\left\langle n_{e}\right\rangle$ are plotted in figure 4 versus the analogously defined relative deviations of the electron temperature $T_{e}$ for a discharge with large fluctuation amplitudes. The data were

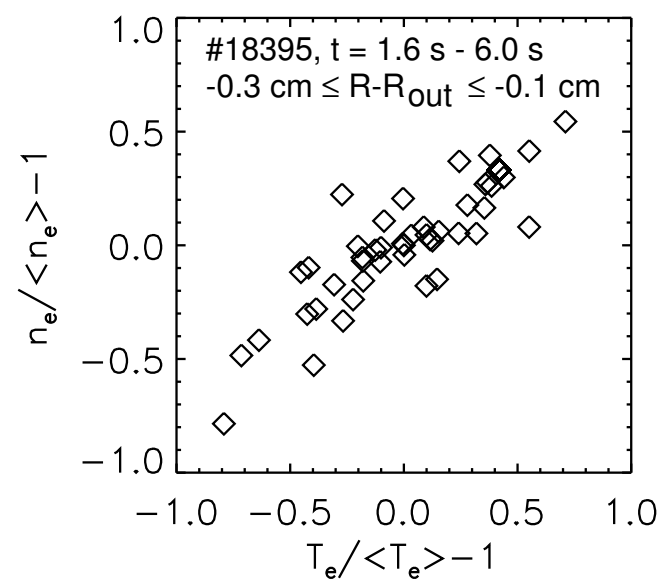

Figure 4: The relative deviations of electron density $n_{e}$ and temperature $T_{e}$, from their mean values $\left\langle n_{e}\right\rangle$ and $\left\langle T_{e}\right\rangle$ averaged for one discharge in time $t$ and over a narrow radial range of $R-R_{\text {out }}$, are well correlated.

evaluated during the plateau phase, and the ELMs were cut out. A clear correlation of the large scale fluctuations of electron density and temperature is found. The 
electron density and temperature data have radial positions $R$ relative to the separatrix at $R_{\text {out }}$ of $-0.3 \mathrm{~cm} \leq R-R_{\text {out }} \leq-0.1 \mathrm{~cm}$. So part of the variation in the data is due to the different radial positions in the edge gradient region where the electron density and temperature data are evaluated. The relative variation of the electron density can be estimated with the gradient scale length $l_{n e}$ of the $1 \mathrm{D}$ radial electron density profile, $\delta n_{e} /\left\langle n_{e}\right\rangle=(\Delta R / 2) / l_{n e}$, and analogously for the electron temperature. The resulting relative variations, $\delta n_{e} /\left\langle n_{e}\right\rangle=0.1$ and $\delta T_{e} /\left\langle T_{e}\right\rangle=0.2$, are so small that the variations observed in figure 4 are dominated by the plasma fluctuations.

Such a high correlation between the electron density and temperature fluctuations may indicate, that the advection by the $\vec{E} \times \vec{B}$ drift is dominating in the transport of both electron density and temperature. In simulations of gyro-fluid turbulence [12] $\vec{E} \times \vec{B}$ advection was indeed found to be dominating in the transport.

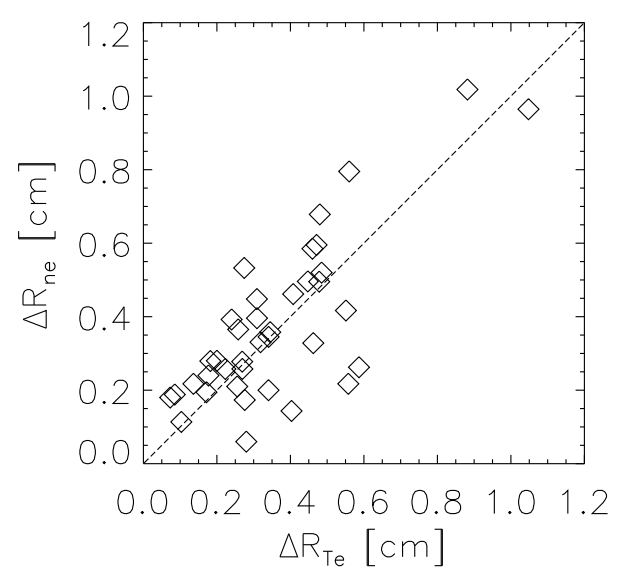

Figure 5: The radial diameters, $\Delta R_{n e}$ and $\Delta R_{\text {Te }}$ of the fluctuation structures of electron density and temperature for several discharges are strongly correlated and are of the same mean size, $\left\langle\Delta R_{n e}\right\rangle /\left\langle\Delta R_{T e}\right\rangle=1.04 \pm 0.14$. (Typical errors of measurement: $\left.\Delta R_{n e} / R_{n e}=10 \%, \Delta R_{T e} / R_{T e}=10 \%\right)$.

The relative fluctuation amplitudes of the electron temperature are usually larger than for the electron density, as can be seen in figure 4, and which is also found in simulations of the edge turbulence [13]. This correlates with the usual finding that the gradient scale lengths $l_{T e}$ of the radial electron temperature profiles are typically smaller than the gradient scale lengths $l_{n e}$ for the electron density profiles [11]. In simulations of drift wave turbulence (see e. g. $[14,15]$ ) it is found that the relative fluctuation amplitude scales with the radial diameter of the fluctuating structure over the gradient scale length of the profile, which for fluctuations of the electron density is $\tilde{n}_{e} / n_{e} \sim \Delta R_{n e} /\left(2 l_{n e}\right)$. The radial diameters of the fluctuating structures in the electron density can be estimated by $\Delta R_{n e}=2 \operatorname{Var}^{1 / 2}\left(n_{e} /\left\langle n_{e}\right\rangle\right) l_{n e}$, where the relative fluctuation level of the electron density was estimated by $\tilde{n}_{e} / n_{e}=$ $\operatorname{Var}^{1 / 2}\left(n_{e} /\left\langle n_{e}\right\rangle\right)$. The radial diameters of the fluctuating structures in the electron density are plotted versus the analogously defined radial diameters of the fluctuating structures in the electron temperature, $\Delta R_{T e}$, in figure 5. The radial diameters $\Delta R_{n e}$ 
and $\Delta R_{T e}$ are correlated, and of the same size: The mean radial diameters are equal within the error of measurement, $\left\langle\Delta R_{n e}\right\rangle /\left\langle\Delta R_{T e}\right\rangle=1.04 \pm 0.14$. It is interesting to note that the radial diameters $\Delta R_{\mathrm{ne}}, \Delta R_{\mathrm{Te}}$ of the fluctuating structures in electron density and temperature agree with the radial widths found in the $2 \mathrm{D}$ snapshots of the electron density and temperature fluctuations [3].

It might be argued that if the fluctuations would be isotropic, the small scale turbulent structures would be averaged out by the large vertical size of the scattering volume, so that fluctuating structures with radial diameters down to $1 \mathrm{~mm}$ should not be observable. By coincidence, however, the vertically elongated scattering volumes appear to be well adapted to the vertically elongated shape of the fluctuating structures existing on the low field side of the tokamak: Indeed fluctuating structures of electron density and temperature exist in large numbers, which fit into the vertically elongated scattering volumes so that relative fluctuation amplitudes of up to $100 \%$ are measured [3].

\subsection{Variation of the large scale fluctuations with electron density}

Although the data set of the relative fluctuation amplitudes of electron density and temperature, $\operatorname{Var}^{1 / 2}\left(n_{e} /\left\langle n_{e}\right\rangle\right)$ and $\operatorname{Var}^{1 / 2}\left(T_{e} /\left\langle T_{e}\right\rangle\right)$, includes plasmas of quite different heating powers, plasma shapes and magnetic fields, a strong correlation of the relative fluctuation amplitudes is found with the electron density. The relative fluctuation amplitudes of electron density and temperature rise with the edge line averaged electron density $\left\langle n_{e}\right\rangle_{H 5}$, and the line averaged electron density $\left\langle n_{e}\right\rangle_{H 1}$. (see figure 6). The same behaviour is also observed for the relative electron pressure, which for correlated electron density and temperature fluctuations is $\Delta p_{e} / p_{e}=\operatorname{Var}^{1 / 2}\left(n_{e} /\left\langle n_{e}\right\rangle\right)+\operatorname{Var}^{1 / 2}\left(T_{e} /\left\langle T_{e}\right\rangle\right)$.

When plotting the relative fluctuation amplitudes versus the local electron density $\left\langle n_{e}\right\rangle$ (see figure 6), the relative fluctuations increase for $2 \times 10^{19} \mathrm{~m}^{-3}<\left\langle n_{e}\right\rangle<$ $4 \times 10^{19} \mathrm{~m}^{-3}$ and decrease for larger local densities $\left\langle n_{e}\right\rangle$ : For higher electron densities, $\left\langle n_{e}\right\rangle>4 \times 10^{19} \mathrm{~m}^{-3}$, the radial positions, where the histogram of the fluctuations are symmetric, are shifted inwards to smaller minor radii, where the amplitudes of the fluctuations get smaller. This also means that for all radial positions further outwards the histograms have a positive skewness. Here it is necessary to distinguish between a native parameter dependence (beta, collisionality, etc), and the strong radial variation of the parallel/perpendicular scale ratio in evaluating such findings [16]. The differing regime of this ratio, which leads to parallel thermal electron motion being comparatively fast in the core while moderate in the edge, relative to turbulence eddy lifetime, is likely responsible for most of the differences between core and edge turbulence.

The plots in figure 6 can also be made with densities normalised to the respective Greenwald densities [17]. The scatter in the data is then, however, not reduced, but slightly larger. In terms of normalised electron densities the relative fluctuations of electron density and temperature are larger than about $5 \%$, so that they become observable, for $\left\langle n_{e}\right\rangle_{H 1} / n_{G W}>0.35$, with $n_{G W}$ as the Greenwald density. The largest 

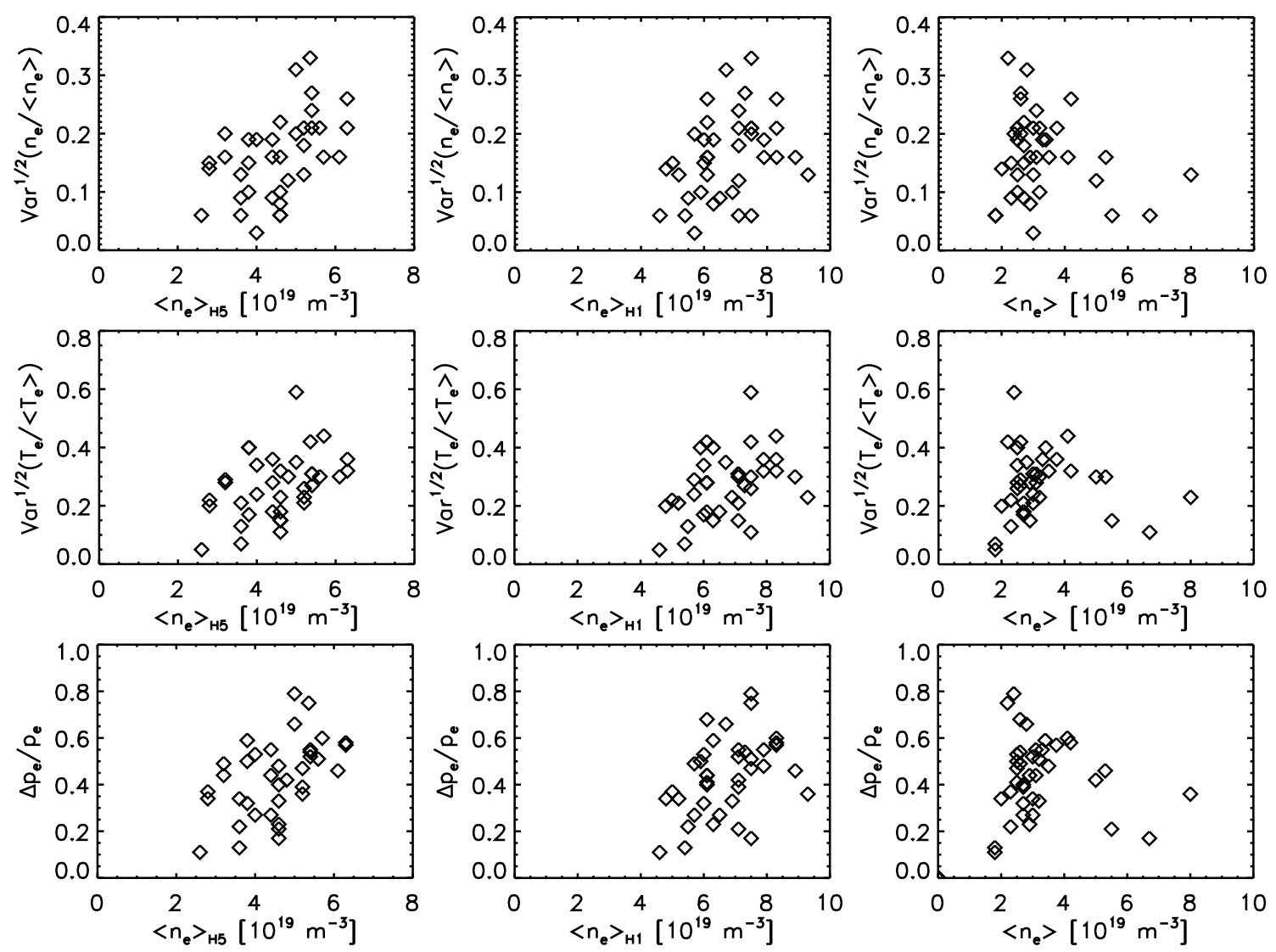

Figure 6: The relative fluctuations of the electron density, $\operatorname{Var}^{1 / 2}\left(n_{e} /\left\langle n_{e}\right\rangle\right)$, temperature, $\operatorname{Var}^{1 / 2}\left(T_{e} /\left\langle T_{e}\right\rangle\right)$, and pressure, $\Delta p_{e} / p_{e}$, increase with rising edge line averaged electron density $\left\langle n_{e}\right\rangle_{H 5}$, and the line averaged electron density $\left\langle n_{e}\right\rangle_{H 1}$. With rising local electron density $\left\langle n_{e}\right\rangle$ the fluctuations first increase and then decrease again: For larger electron densities the positions of the symmetric histograms are so far inwards that the fluctuations decrease again. (Typical errors of measurement: $\left.\Delta\left(\left\langle n_{e}\right\rangle_{H 1}\right) /\left\langle n_{e}\right\rangle_{H 1}=3 \%, \Delta\left(\left\langle n_{e}\right\rangle_{H 5}\right) /\left\langle n_{e}\right\rangle_{H 5}=3 \%, \Delta\left\langle n_{e}\right\rangle /\left\langle n_{e}\right\rangle<5 \%\right)$.

line averaged densities $\left\langle n_{e}\right\rangle_{H 1}$ in figure 6 correspond to about $90 \%$ Greenwald density.

\subsection{Variation of perpendicular electron heat diffusivity and neutral particle flux with the amplitudes of the electron density and temperature fluctuations}

Another question is, whether the fluctuations, which are observed in the pedestal, result in increased perpendicular transport.

In the following it is assumed that mainly the electron heat conductivity determines the perpendicular heat transport. The perpendicular electron heat diffusivity $\chi_{\perp}$ in the pedestal can then be determined from the perpendicular heat flux, $P_{\text {heat }}-P_{\text {rad }}=A\left\langle n_{e}\right\rangle \chi_{\perp} d\left\langle T_{e}\right\rangle / d R$, where $P_{\text {heat }}=P_{O H}+P_{N B I}+P_{I C R H}$ is the total applied heating power with $P_{O H}$ as the ohmic heating power and $P_{\text {rad }}$ as the power 

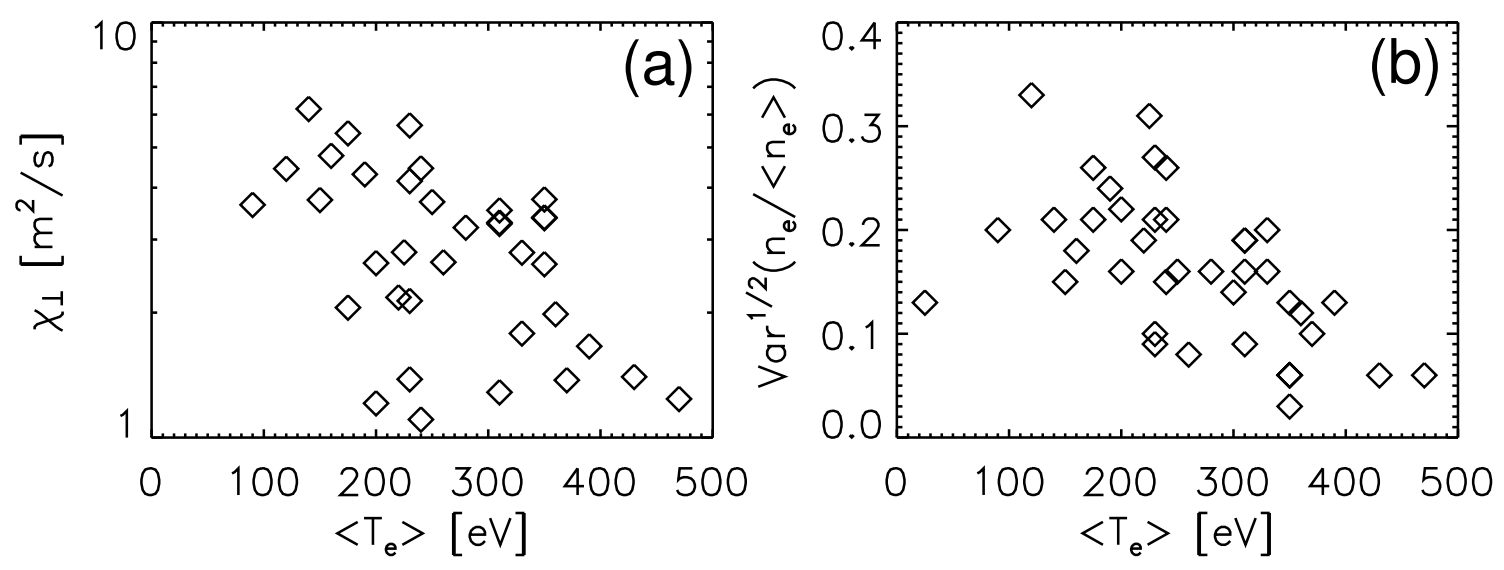

Figure 7: The perpendicular electron heat diffusivity $\chi_{\perp}$ in the pedestal (a) decreases with increasing local electron temperature $\left\langle T_{e}\right\rangle$, which is averaged over the time interval of evaluation. The relative amplitudes of the fluctuations of the electron density $\operatorname{Var}^{1 / 2}\left(n_{e} /\left\langle n_{e}\right\rangle\right)(b)$ also decrease with rising electron temperature $\left\langle T_{e}\right\rangle$. (Typical errors of measurement: $\left.\Delta \chi_{\perp} / \chi_{\perp}=15 \%, \Delta\left\langle T_{e}\right\rangle /\left\langle T_{e}\right\rangle=7 \%\right)$.

lost by radiation, which is determined by bolometry. The local electron density $\left\langle n_{e}\right\rangle$ and electron temperature gradient $d\left\langle T_{e}\right\rangle / d R$ are taken from the time averaged background profiles containing the large scale fluctuations and are measured by Thomson scattering. The local heat flux is assumed to extend over an area of $A \approx 10 \mathrm{~m}^{2}$ of the plasma surface on the low field side, which is about a quarter of the total plasma surface at the separatrix. With increasing electron temperature $\left\langle T_{e}\right\rangle$, which is an average over the time interval of evaluation, the perpendicular electron heat diffusivity decreases (see figure $7(\mathrm{a})$ ). The relative amplitudes of the electron density fluctuations decrease also with rising electron temperature (see figure $7(\mathrm{~b})$ ). A slight increase in the perpendicular electron heat diffusivity $\chi_{\perp}$ is found with increasing relative amplitudes of the fluctuations of electron density and temperature when making a linear fit to the data (see figure 8). The scatter of the data is much larger than would be expected from the errors of measurement of the perpendicular electron heat diffusivity $\chi_{\perp}$, which shows up in the empirical correlation coefficients of only $\rho\left[\chi_{\perp}, \operatorname{Var}^{1 / 2}\left(n_{e} /\left\langle n_{e}\right\rangle\right)\right]=0.32$ and $\rho\left[\chi_{\perp}, \operatorname{Var}^{1 / 2}\left(T_{e} /\left\langle T_{e}\right\rangle\right)\right]=0.25$. This indicates that the perpendicular electron heat diffusivity $\chi_{\perp}$ does not depend directly on the relative amplitudes of the fluctuations of electron density, or temperature, but better suited scaling parameters are needed.

A non-negligible recycling flux has been observed in the main chamber of diverted tokamaks especially during $\mathrm{H}$ mode $[18,19,20]$. It was suggested that this flux is the result of a direct radial ion flux to the wall causing a major part of the main neutral recycling. The origin of the radial ion flux in the SOL is thought to be due to turbulence.

With our data an increase of the particle transport with the relative fluctuation amplitudes of the electron density and temperature in the pedestal is found when looking at the neutral particle flux. The neutral particle flux in the outer midplane, $f_{m p l}$, increases with the relative electron density fluctuations $\operatorname{Var}^{1 / 2}\left(n_{e} /\left\langle n_{e}\right\rangle\right)$ in the 

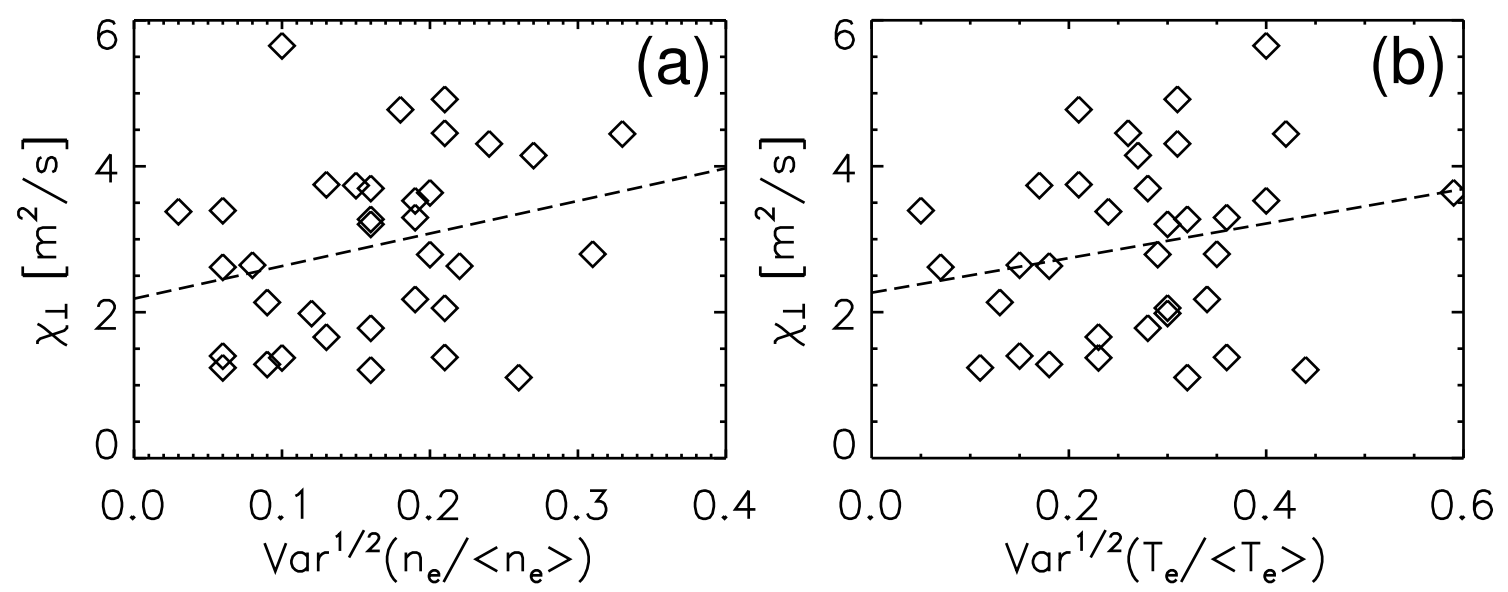

Figure 8: The perpendicular electron heat diffusivity $\chi_{\perp}$ in the pedestal increases slightly with increasing amplitudes of the fluctuations of electron density $\operatorname{Var}^{1 / 2}\left(n_{e} /\left\langle n_{e}\right\rangle\right)$ (a) and temperature $\operatorname{Var}^{1 / 2}\left(T_{e} /\left\langle T_{e}\right\rangle\right)$ (b) as indicated by the linear fit to the data points. The empirical correlation coefficients are, however, low, $\rho\left[\chi_{\perp}, \operatorname{Var}^{1 / 2}\left(n_{e} /\left\langle n_{e}\right\rangle\right)\right]=0.32$ (a) and $\rho\left[\chi_{\perp}, \operatorname{Var}^{1 / 2}\left(T_{e} /\left\langle T_{e}\right\rangle\right)\right]=0.25$ (b). (Typical error of measurement: $\left.\Delta \chi_{\perp} / \chi_{\perp}=15 \%\right)$.

pedestal (figure 9(a)). The number of the data points shown in figure 9 is smaller than in the previous figures, because the neutral particle flux $f_{m p l}$ is not available for each investigated discharge. It could be argued that since the relative fluctuations in the pedestal increase with the plasma density (figure 6) and typically an increase of the neutral particle flux with plasma density is observed [21] that the correlation of the neutral particle flux and the relative fluctuations in the pedestal is a cocorrelation. For comparison also the neutral particle flux in the pumping chamber, $f_{p c h}$, is plotted versus the relative electron density fluctuations in figure 9 (b). But here less correlation is found. The behaviour of the two ionisation gauges, which measure the fluxes $f_{m p l}$ and $f_{p c h}$, to the plasma density is different: The neutral particle flux in the midplane, $f_{m p l}$, is less correlated to the plasma density $\left\langle n_{e}\right\rangle_{H 1}$ : The maximum neutral fluxes in the midplane are not found for the largest plasma densities $\left\langle n_{e}\right\rangle_{H 1}$ (figure 9(c)). The neutral particle flux in the pumping chamber, $f_{p c h}$, is well correlated to the plasma density and behaves as usually expected [21]. So the good correlation of the neutral particle flux in the outer midplane, $f_{m p l}$, with the fluctuations in the pedestal is with high probability due to the high sensitivity of this ionisation gauge to the particle fluxes induced by the fluctuations in the pedestal.

\section{Comparison with scalings of drift-Alfvén tur- bulence}

We now compare the measured fluctuation quantities to elements of drift Alfvén turbulence. A detailed comparison with simulations is not foreseen, because the 

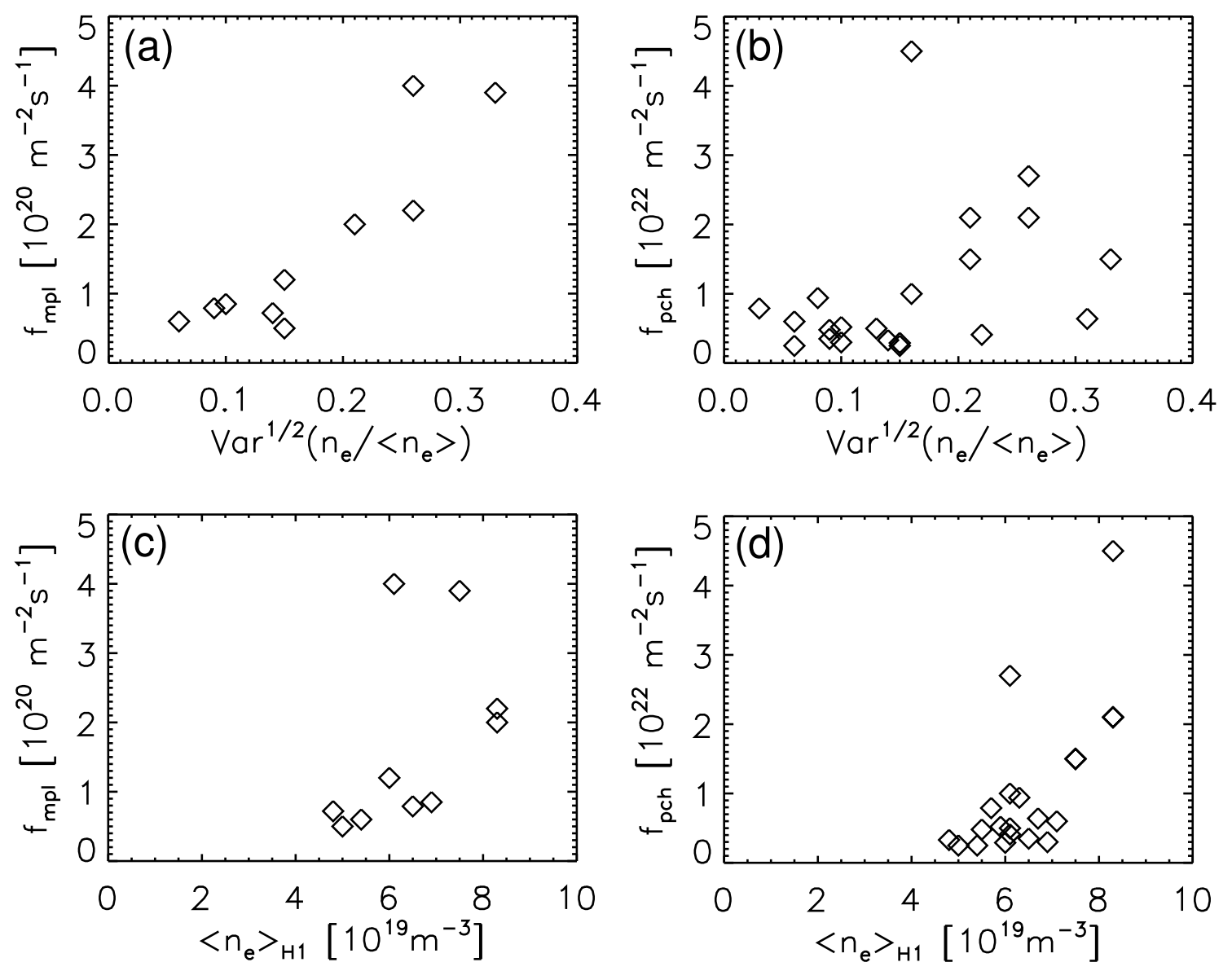

Figure 9: The neutral gas flux in the outer midplane, $f_{m p l}$, increases with rising electron density fluctuations, $\operatorname{Var}^{1 / 2}\left(n_{e} /\left\langle n_{e}\right\rangle\right)$ (a), but does not show the largest fluxes for the largest densities $\left\langle n_{e}\right\rangle_{H 1}(c)$. This is different to the neutral gas flux in the pumping chamber, $f_{\text {pch }}$, showing less correlation with the fluctuations in the pedestal (b) and a better correlation with the plasma density (d). (Typical errors of measurement: $\left.\Delta f_{m p l} / f_{m p l}=10 \%, \Delta f_{p c h} / f_{p c h}=10 \%\right)$.

latter have not adequately explained the phenomenology of $\mathrm{H}$ mode fluctuations [13, 16]. Indeed the drift Alfvén turbulence, especially the higher-beta version in which longer wavelengths are nonlinearly driven, is precisely what must be eliminated to produce the $\mathrm{H}$ mode. (The hypothesis is of remnant drift wave turbulence if suppression of the longer wavelengths caused by the ion dynamics is what causes the $\mathrm{H}$ mode, recalling that electron heat transport in the $\mathrm{H}$-mode is still strongly anomalous.) However, it is of interest to know whether these parameter scalings are relevant strictly on empirical grounds. The parameters which enter in drift Alfvén turbulence are summarised in table 2 . 
Table 2: Parameters in drift Alfvén turbulence (see e. g. [7]).

\begin{tabular}{l|l}
\hline drift wave collisionality & $C=0.51\left[\nu_{e} /\left(c_{S} / l_{\perp}\right)\right]\left(m_{e} / m_{i}\right)\left(q R_{0} / l_{\perp}\right)^{2}$ \\
magnetic curvature parameter & $\omega_{B}=2 l_{\perp} / R_{0}$ \\
drift Alfvén parameter & $\hat{\beta}=\left(\mu_{0} p_{e} / B_{t}^{2}\right)\left(q R_{0} / l_{\perp}\right)^{2}$ \\
normalised mass ratio & $\hat{\mu}=\left(m_{e} / m_{i}\right)\left(q R_{0} / l_{\perp}\right)^{2}$ \\
ion sound gyro-radius & $\rho_{S}=c_{S} / \Omega_{i}$ \\
scale length of collisional limit of & $\Delta_{d}=C^{1 / 2} \rho_{S}$ \\
drift waves & \\
scale length of resistive ballooning & $L_{0}=2 \pi C^{1 / 2} \omega_{B}^{1 / 4} \rho_{S}$ \\
collisionless skin depth & $\sigma_{0}=(\hat{\mu} / \hat{\beta})^{1 / 2} \rho_{S}$ \\
electron ion collision frequency $[22]$ & $\nu_{e}=(4 / 3)\left[\ln (\Lambda) e^{4} /\left(4 \pi \epsilon_{0}\right)^{2}\right] n_{e}\left[2 \pi /\left(m_{e} T_{e}^{3}\right)\right]^{1 / 2}$ \\
ion sound speed & $c_{S}=\left(T_{e} / m_{i}\right)^{1 / 2}$ \\
generalised profile scale length & $l_{\perp}=l_{p e}$ \\
electron, ion mass (deuterium) & $m_{e}, m_{i}$ \\
ion cyclotron frequency & $\Omega_{i}$ \\
magnetic constant & $\mu_{0}$ \\
electric constant & $\epsilon_{0}$ \\
elementary charge & $e$ \\
Coulomb logarithm & $\ln (\Lambda)=20$ \\
safety factor & $q$ \\
major radius & $R_{0}=1.65 m$ \\
\hline
\end{tabular}

\subsection{Parameter space}

The drift Alfvén parameter $\hat{\beta}$, measuring the magnetic induction, the normalised mass ratio $\hat{\mu}$, important for Landau damping, and the drift wave collisionality $C$ are shown for the plasmas of the data base in figure 10. The plasma parameters were varied such that no clear correlation can be found between the drift Alfvén parameter $\hat{\beta}$ and the drift wave collisionality $C$ (see figure 10(a)). The drift Alfvén parameter $\hat{\beta}$ is typically of the order of the normalised mass ratio $\hat{\mu}$ (see figure $10(\mathrm{~b})$ ). This means that the collisionless skin depth $\sigma_{0}=(\hat{\mu} / \hat{\beta})^{1 / 2} \rho_{S}$ is for the investigated $\mathrm{H}$ mode edge plasmas of the order of the ion sound gyro-radius $\rho_{S}$. Similar to core turbulence, the collisionless skin depth $\sigma_{0}$ replaces the ion sound gyro-radius $\rho_{S}$ as the smallest relevant scale length whenever $\hat{\beta}>\hat{\mu}$ (i.e., the electron dynamical beta $\mu_{0} p_{e} / B_{t}^{2}$ becomes larger than $\left.m_{e} / m_{i}\right)$.

\subsection{Electron pressure fluctuations}

Simple mixing length theory suggests the fluctuation amplitude should scale with the scale length ratio of the fluctuations to the background profile. If the fluctuation scale length is taken as $\rho_{S}$ times a constant independent of parameters, what is referred to as gyro-Bohm scaling results (see the dashed line in figure 11). However, with dependence on other dimensionless parameters such as $\hat{\beta}$ and $C$, deviations should occur. The results in figure 11 show strong deviations, when all data are used 

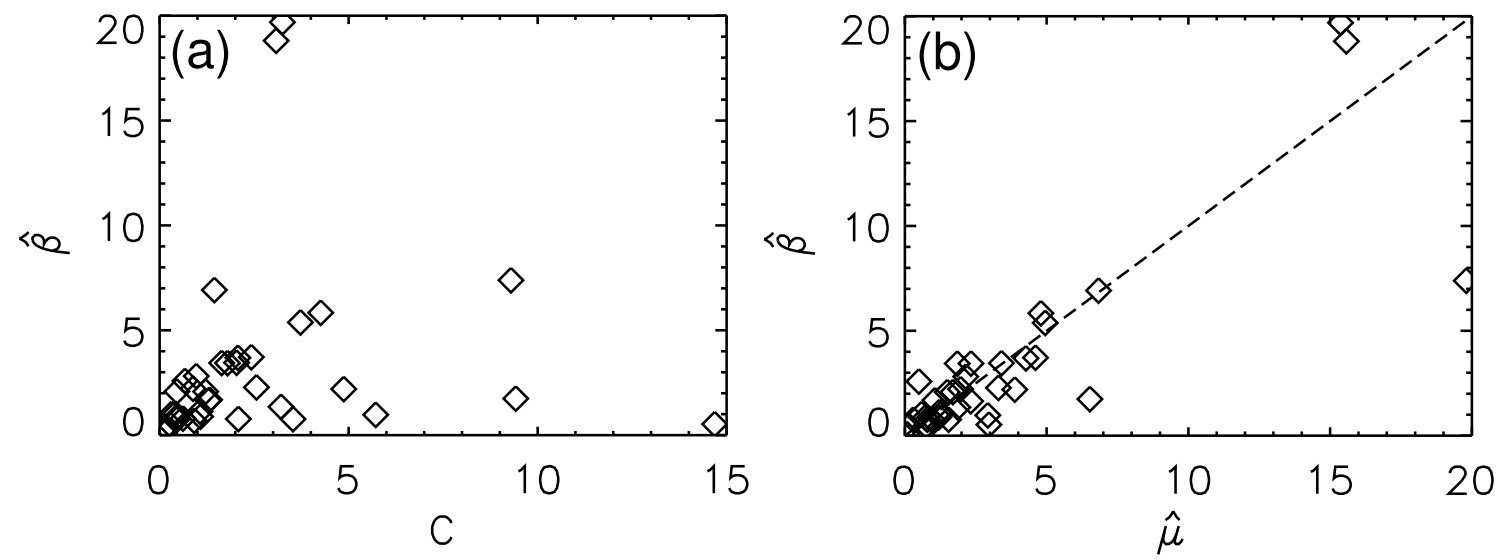

Figure 10: The drift wave collisionalities $C$ and the drift Alfvén parameters $\hat{\beta}$ of the investigated plasmas are not correlated (a). The drift Alfvén parameter $\hat{\beta}$ is typically of the order of the normalised mass ratio $\hat{\mu}(b)$, so that the collisionless skin depth $\sigma_{0}$ is of the order of the ion sound gyro-radius $\rho_{S}$.

(figure 11(a)). If only higher values of the collisionality, $C \geq 2$, or of beta, $\hat{\beta} \geq 2$, are allowed (figures 11(b) and (c) respectively), we find that stronger collisionality causes deviations from the gyro-Bohm scaling, while higher $\hat{\beta}$ places the points closer to the line. While simulations have yet to explain this result, it indicates that both the $\mathrm{H}$ mode phase and the spatial region closer to the core has turbulence, which is more electrostatic in character and hence more gyro-Bohm like. However, the use of differing spatial regions encounters the problem that the profile scale length and hence the parallel/perpendicular scale ratio $q R / l_{\perp}$ varies strongly in this region [16]. Further experimental investigations will be necessary to definitively separate the beta dependence from the scale ratio dependence.

\subsection{Radial scale lengths of electron density fluctuations}

In drift Alfvén turbulence different phenomena act on different scale lengths, which are resolved in the simulations: the ion sound gyro-radius $\rho_{S}$, the collisional limit of drift waves $\Delta_{d}$, the scale length of resistive ballooning $L_{0}$, and the collisionless skin depth $\sigma_{0}$. These scale lengths are defined in table 2. In the following the inferred radial diameters of the electron density fluctuations $\Delta R_{n e}$ are normalised to $\rho_{S}$ and are compared to these scale lengths, which are normalised accordingly. The inferred normalised radial diameters $\Delta R_{n e} / \rho_{S}$ are proportional to the relative amplitude of the density fluctuations $\operatorname{Var}^{1 / 2}\left(n_{e}\right)$, and to the density gradient scale length normalised to the ion sound gyro-radius, $l_{n e} / \rho_{S}$. For the inferred radial diameters $\Delta R_{n e}$ there is a variation in the relative amplitudes of the electron density fluctuations, $0.03 \leq \operatorname{Var}^{1 / 2}\left(n_{e}\right) \leq 0.33$ (see figure 6), and in the normalised gradient scale lengths of the electron density, $33 \leq l_{n e} / \rho_{S} \leq 5.0 \times 10^{2}$. So the different values, which are found for the inferred radial diameter $\Delta R_{n e}$, are not due to the variation of only a single parameter.

The inferred radial diameters of the electron density fluctuations $\Delta R_{n e}$ correlate 

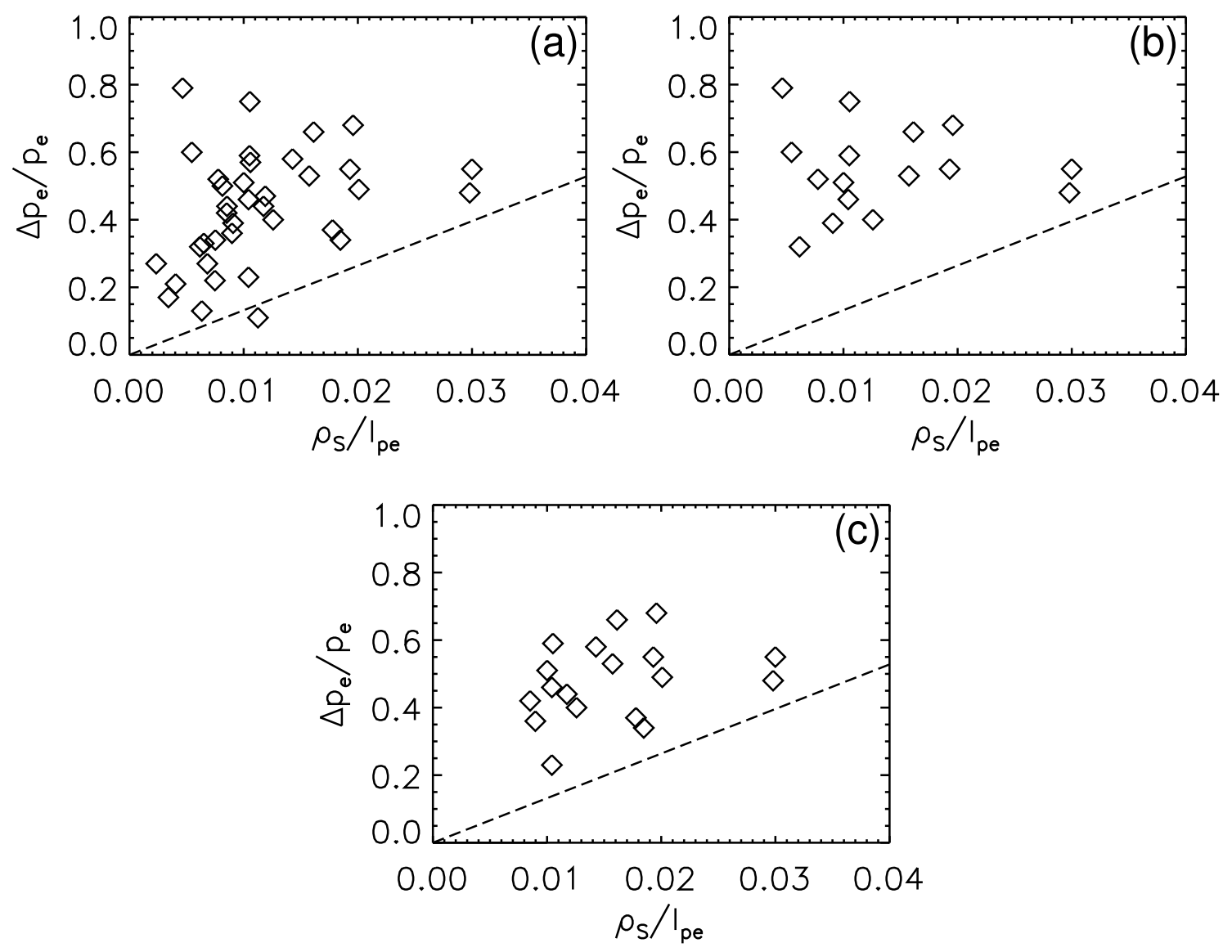

Figure 11: The relative electron pressure fluctuations $\Delta p_{e} / p_{e}$ show strong deviations from Gyro-Bohm scaling results (dashed line), if all data are used (a). Data for higher collisionality, $C \geq 2$, show stronger deviations from Gyro-Bohm scaling (b), while data with higher beta, $\hat{\beta} \geq 2$, are closer to Gyro-Bohm scaling (c). (Typical errors of measurement: $\left.\delta\left(\Delta p_{e} / p_{e}\right) /\left(\Delta p_{e} / p_{e}\right) \leq 8 \%, \Delta\left(\rho_{S} / l_{p e}\right) /\left(\rho_{S} / l_{p e}\right) \leq 18 \%\right)$.

already quite well with the scale length of the collisional limit of drift waves $\Delta_{d}$ (figure 12(a)) and with the scale length of resistive ballooning $L_{0}$ (figure 12(b)), but not with the collisionless skin depth $\sigma_{0}$ (figure 12(c)). An even better correlation of the inferred radial diameters of the electron density fluctuations $\Delta R_{n e}$, especially for large values of $\Delta R_{n e} / \rho_{S}$, is obtained for the empirically constructed scale length $\Delta_{h}=\hat{\beta}^{1 / 2} C^{1 / 2} \omega_{B}^{1 / 2} \rho_{S}$, which is a hybrid scale length between ideal and resistive MHD. The ideal MHD part, $\hat{\beta}^{1 / 2} \omega_{B}^{1 / 4}$, tends to enlarge the spatial diameters of the fluctuating structures [7]. So in the $\mathrm{H}$ mode edge, dynamics at the large ideal and resistive MHD scales plays a role in steering the smaller scale electron turbulence, but the latter still carries the transport.

\subsection{Scale lengths of perpendicular electron heat diffusivity}

The spatial scales for the perpendicular electron heat diffusivity $\chi_{\perp}$ are investigated in this section. For this the perpendicular electron heat diffusivity $\chi_{\perp}$ normalised 

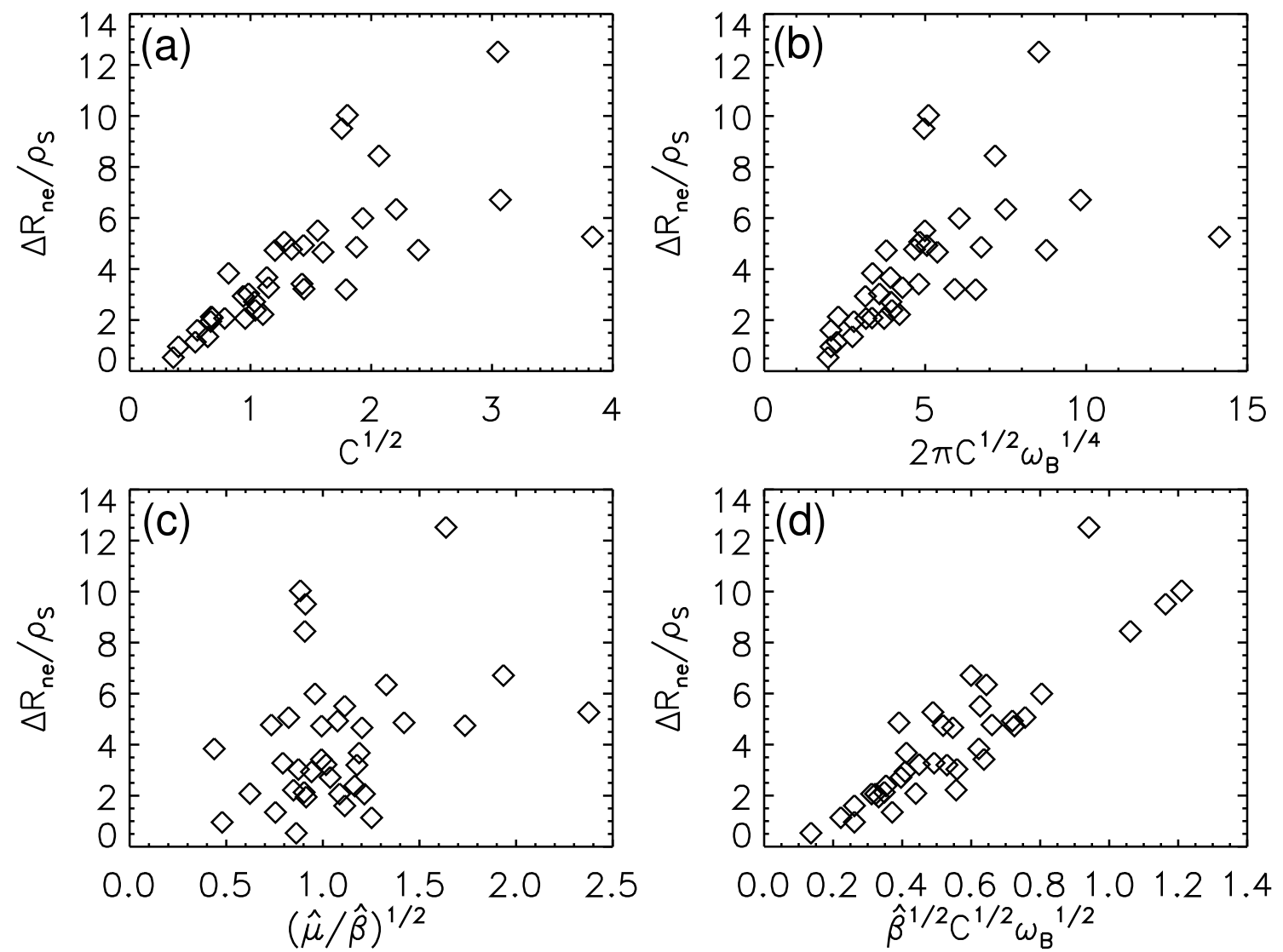

Figure 12: The radial scale lengths $\Delta R_{n e}$, which are normalised to $\rho_{S}$, are compared to the accordingly normalised scale lengths of the collisional limit of driftwaves, $\Delta_{d} / \rho_{S}=C^{1 / 2}(a)$, of resistive ballooning, $L_{0} / \rho_{S}=2 \pi C^{1 / 2} \omega_{B}^{1 / 4}(b)$, and of the collisionless skin depth, $\sigma_{0} / \rho_{S}=(\hat{\mu} / \hat{\beta})^{1 / 2}(c)$. The best ordering, however, is found for the empirically constructed hybrid scale length between ideal and resistive $M H D, \Delta_{h} / \rho_{S}=\hat{\beta}^{1 / 2} C^{1 / 2} \omega_{B}^{1 / 2},(d)$. (Typical errors of measurement: $\delta\left(\Delta R_{n e} / \rho_{S}\right) /\left(\Delta R_{n e} / \rho_{S}\right)=15 \%, \delta\left(\Delta_{d} / \rho_{S}\right) /\left(\Delta_{d} / \rho_{S}\right)=10 \% \Delta\left(L_{0} / \rho_{S}\right) /\left(L_{0} / \rho_{S}\right)=$ $\left.15 \%, \Delta\left(\sigma_{0} / \rho_{S}\right) /\left(\sigma_{0} / \rho_{S}\right)=9 \%, \delta\left(\Delta_{h} / \rho_{S}\right) /\left(\Delta_{h} / \rho_{S}\right)=20 \%\right)$.

to the usually used Gyro-Bohm diffusivity, $D_{G B}=\left(\rho_{S} / a\right) T_{e} /\left(16 e B_{t}\right)$, with $a=$ $0.5 \mathrm{~m}$ as the minor radius of the plasma, is used. Good ordering of the normalised perpendicular electron heat diffusivities $\chi_{\perp} / D_{G B}$ is found for the scale lengths of the collisional limit of drift waves $\Delta_{d}$ (figure 13(a)), and for the scale lengths of resistive ballooning $L_{0}$ (figure 13(b)). This was also found in theoretical simulations: The energy transport scales in the same way for both collisional drift wave, and resistive ballooning turbulence [13]. There is, however, increasing scatter of the normalised heat diffusivity towards the lower values of the scale lengths in figures 13(a) and (b). When plotting the normalised perpendicular electron heat diffusivity $\chi_{\perp} / D_{G B}$ versus the collisionless skin depth $\sigma_{0}$ a quite good correlation is found also for the small collisionless skin depths $\sigma_{0}<\rho_{S}$ (figure 13(c)). Only a very small correlation is found between the normalised perpendicular electron heat diffusivity and the hybrid scale between ideal and resistive $\mathrm{MHD}, \Delta_{h}$ (figure $13(\mathrm{~d})$ ), which is the scale length 

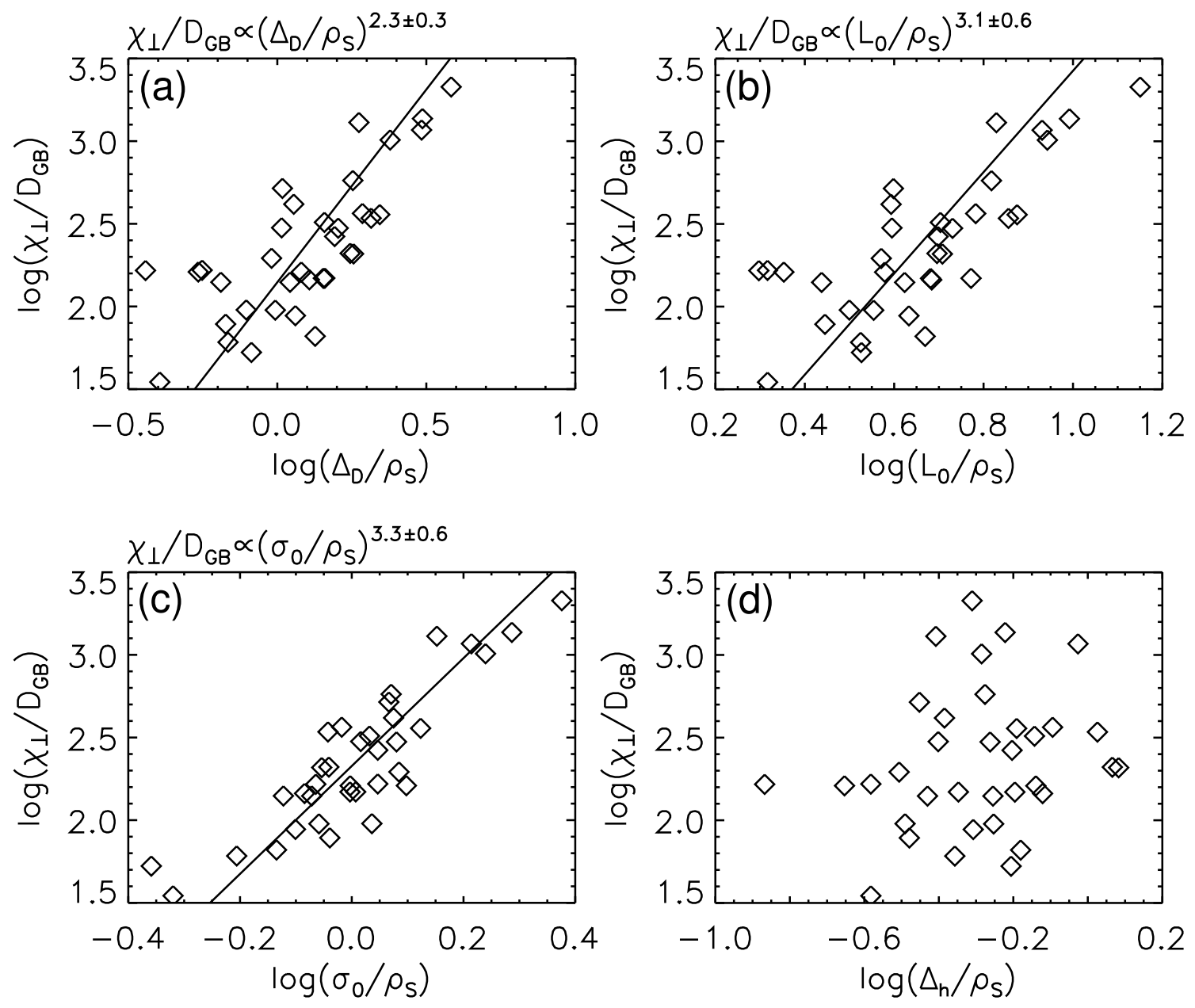

Figure 13: The perpendicular electron heat diffusivity normalised to Gyro-Bohm diffusivity, $\chi_{\perp} / D_{G B}$, correlates quite well with the scale lengths of the collisional limit of drift waves (a), of resistive ballooning (b), and also with the collisionless skin depth (c), but not with the hybrid scale length between ideal and resistive MHD (d). In the fits $\chi_{\perp} / D_{G B} \sim\left(x / \rho_{S}\right)^{a(x)}$ to the data the exponents a $(x)$ for the scale lengths $x$ are the largest for (b) and (c). Apparently both collisional, and collisionless effects are equally strong in $H$ mode edge turbulence. (Typical errors of measurement: $\Delta\left(\chi_{\perp} / D_{G B}\right) /\left(\chi_{\perp} / D_{G B}\right)=20 \%, \delta\left(\Delta_{d} / \rho_{S}\right) /\left(\Delta_{d} / \rho_{S}\right)=10 \%, \Delta\left(L_{0} / \rho_{S}\right) /\left(L_{0} / \rho_{S}\right)=$ $\left.15 \%, \Delta\left(\sigma_{0} / \rho_{S}\right) /\left(\sigma_{0} / \rho_{S}\right)=9 \%, \delta\left(\Delta_{h} / \rho_{S}\right) /\left(\Delta_{h} / \rho_{S}\right)=20 \%\right)$. 
of the radial diameters of the electron density and temperature fluctuations (figure $12(\mathrm{~d}))$. This also explains, why in the plots of the perpendicular electron heat diffusivity versus the relative electron density, or temperature fluctuations (figure 8), only poor correlations are found. The dependency of the perpendicular electron heat diffusivity on the different scale lengths is also seen in the exponents $a(x)$ of the fits $\chi_{\perp} / D_{G B} \sim\left(x / \rho_{S}\right)^{a(x)}$ to the data for the scale lengths $x \in\left\{\Delta_{d}, L_{0}, \sigma_{0}\right\}$ (see figures 13(a)-(c)). The largest exponents are found for resistive ballooning, $a\left(L_{0}\right)=3.1 \pm 0.6$, and for the collisionless skin depth, $a\left(\sigma_{0}\right)=3.3 \pm 0.6$.

For the parameters of the $\mathrm{H}$ mode edge the smallest scale is not always the ion sound gyro-radius $\rho_{S}$, but also the collisionless skin depth $\sigma_{0}$, which is of the same size, or smaller. In this respect the energy transport takes place on the smallest scales of the turbulence.

\section{Summary and conclusion}

Large scale inter-ELM fluctuations of electron density and temperature were investigated by Thomson scattering in the hot pedestal. The histograms of the fluctuation amplitudes of electron density and temperature are symmetric at a radial position, which is roughly in the middle of the edge pedestal. Further inwards the histograms show negative skewness, further outwards the skewness is positive. The actual radial positions of the symmetric histograms move inwards, away from the separatrix when the gradient lengths of the electron temperature, or electron pressure, which show the steepest gradients, get larger. The fluctuations of electron density and temperature are in phase and are observable above a local electron density $n_{e}>2 \times 10^{19} \mathrm{~m}^{-3}$, which corresponds to a Greenwald fraction of the line-averaged electron density of $\left\langle n_{e}\right\rangle_{H 1} / n_{G W}>0.35$. The relative amplitudes of the electron density and temperature fluctuations increase with rising electron density up to $n_{e} \approx 4 \times 10^{19} \mathrm{~m}^{-3}$. Above the local electron density $n_{e}>4 \times 10^{19} \mathrm{~m}^{-3}$ the gradients of the electron density and temperature profiles flatten and the radial positions of the symmetric histograms of the fluctuations move further inwards away from the separatrix. This behaviour is not directly correlated with the Greenwald fraction, because symmetric histograms of the fluctuations with the highest Greenwald fractions $\left(\left\langle n_{e}\right\rangle_{H 1} / n_{G W} \approx 0.9\right)$ are also found closer to the separatrix.

The relative fluctuation amplitudes of electron density and temperature in the pedestal and the neutral particle flux in the outer midplane are correlated. This may indicate that the fluctuations in the pedestal are connected to the particle transport in the SOL.

The radial diameters of the fluctuating structures of electron density and temperature, which are observed in-between ELMs, scale with the hybrid scale between ideal and resistive MHD, $\Delta_{h}$. The ideal MHD part gives the large fluctuation structures, which are observed in the electron density and temperature. This hybrid scale length $\Delta_{h}$ for the fluctuations of electron density and temperature has up to now not yet been seen in simulations of turbulence and thus may stimulate further theoretical investigations. 
While the dynamics of ideal and resistive MHD are found experimentally in the

scale lengths of the electron density and temperature fluctuations, the heat transport by the electrons takes place on smaller scales down to the collisionless skin depth $\sigma_{0}$.

\section{References}

[1] Wagner F et al. 1982491408

[2] Suttrop W 200042 A1

[3] Kurzan B, Horton L D, Murmann H, Neuhauser J, Suttrop W and ASDEX Upgrade Team 200749825

[4] Kurzan B, et al. and ASDEX Upgrade Team 200042237

[5] LaBombard B et al. and Alcator C-Mod Team 2005 Nucl. Fusion 451658

[6] Dudson B D et al. and the MAST Team 200850124012

[7] Scott B 200345 A385

[8] Murmann H, Götsch S, Röhr H, Salzmann H, Steuer K H 1992634941

[9] Kurzan B, Jakobi M, Murmann H, ASDEX Upgrade Team 200446299

[10] Haas G, Bosch H S 1998 Vacuum 5139

[11] Neuhauser J et al. and ASDEX Upgrade Team 200244855

[12] Scott B D 2005 Phys. Plasmas 12082305

[13] Scott B D 200749 S25

[14] Scott B D 1992 Phys. Fluids 42468

[15] Guzdar P N, Drake J F, McCarthy D, Hassam A B, Liu C S 1993 Phys. Fluids 53712

[16] Scott B D 2000 Phys. Plasmas 71845

[17] Greenwald M 200244 R27

[18] Neu R et al. and ASDEX Upgrade Team 2002441021

[19] LaBombard B et al. and Alcator Group 2000 Nucl. Fus. 402041

[20] Stangeby P C 2002 Phys. Plasmas 93489

[21] Scarabosio A, Haas G, Müller H W, Pugno R, Wischmeier M, ASDEX Upgrade Team 2009 J. Nucl. Mater. at press, doi:10.1016/j.jnucmat.2009.01.057

[22] Braginskii S I 1965 Rev. Plasma Phys. 1205 